Purdue University

Purdue e-Pubs

Other Nanotechnology Publications

Birck Nanotechnology Center

7-1-2007

\title{
Coupled mode space approach for the simulation of realistic carbon nanotube field-effect transistors
}

\author{
Gianluca Fiori \\ University of Pisa \\ Giuseppe Iannaccone \\ University of Pisa \\ Gerhard Klimeck \\ School of Electrical and Computer Engineering and Birck Nanotechnology Center, Purdue University, gekco@purdue.edu
}

Follow this and additional works at: http://docs.lib.purdue.edu/nanodocs

Fiori, Gianluca; Iannaccone, Giuseppe; and Klimeck, Gerhard, "Coupled mode space approach for the simulation of realistic carbon nanotube field-effect transistors" (2007). Other Nanotechnology Publications. Paper 32.

http://docs.lib.purdue.edu/nanodocs/32

This document has been made available through Purdue e-Pubs, a service of the Purdue University Libraries. Please contact epubs@purdue.edu for additional information. 


\title{
Coupled Mode Space Approach for the Simulation of Realistic Carbon Nanotube Field-Effect Transistors
}

\author{
Gianluca Fiori, Giuseppe Iannaccone, and Gerhard Klimeck, Senior Member, IEEE
}

\begin{abstract}
A coupled mode space approach within the nonequilibrium Green's function formalism is presented, which allows to perform simulations of realistic carbon nanotube field-effect transistors (CNT-FETs) with no spatial symmetry. Computing time is significantly reduced with respect to the real space approach, since only few modes are needed in order to obtain accurate results. The advantage of the method increases with increasing nanotube diameter, and is a factor of 20 in computing time for a $(25,0)$ nanotube. As a consequence, computationally demanding simulations like those required by a statistical investigation, or by a device performance study based on the exploration of the design space, become more affordable. As a further test of the method, we have applied the coupled mode space approach to double-gate CNT-FETs devices and devices with discrete distribution of doping atoms. In the latter case, nonballistic transport due to elastic scattering with ionized impurities in doped source and drain extensions occurs. We show that even in the case of very rough potential, the coupled mode space approach is accurate with very few modes, enabling atomistic simulations of statistical properties with reduced computational resources.
\end{abstract}

Index Terms-Carbon nanotube, mode space, nonequilibrium Green's function (NEGF), three-dimensional simulations, tightbinding Hamiltonian.

\section{INTRODUCTION}

$\mathbf{C}$ ARBON nanotube field-effect transistors (CNT-FETs) are promising candidates to satisfy the requirements at the end of the ITRS. However, fabrication technology still poses serious challenges, such as the selection of metallic and semiconducting carbon nanotubes, the poor control of the contact quality, and the large diameter dispersion. In this scenario, adequate device simulation tools are necessary to interpret experimental results, and to provide device guidelines for the fabrication of CNTFETs. In particular, the exploration of the design space requires agile and fast tools.

Manuscript received September 27, 2006; revised January 29, 2007. This work was supported in part by the EU SINANO NoE under Contract 506844 and in part by the European Science Foundation EUROCORES Programme Fundamentals of Nanoelectronics, through fundings from Consiglio Nazionale delle Ricerche (awarded to IEEIIT-PISA) and the European Commission Sixth Framework Programme under Project Dewint (Contract ERAS-CT-2003-980409). The work of G. Klimeck was supported by the National Science Foundation under Grant EEC-0228390. The review of this paper was arranged by Associate Editor D. Frank.

G. Fiori is with the Dipartimento di Ingegneria dell'Informazione, Informatica, Elettronica, Telecomunicazioni, University of Pisa, 56120 Pisa, Italy.

G. Iannaccone is with the Dipartimento di Ingegneria dell'Informazione, Informatica, Elettronica, Telecomunicazioni, University of Pisa, Italy, and also with IEIIT-CNR, I-56122 Pisa, Italy.

G. Klimeck is with the School of Electrical and Computer Engineering, Purdue University, West Lafayette, IN 47907 USA.

Color versions of one or more of the figures in this paper are available online at http://ieeexplore.ieee.org.

Digital Object Identifier 10.1109/TNANO.2007.896842
To this purpose, an effective mass approach, whose validity has been demonstrated in [1], has been followed in [2] providing results in quantitative agreement with more accurate band models based on a tight-binding description and Huckel theory. However, as far as short devices are concerned, atomistic fully quantum mechanical approaches are needed in order to accurately reproduce all the relevant electrical quantities. The nonequilibrium Green's function formalism (NEGF) in particular has shown great capabilities in the simulation of quantum transport in nanoscale devices [3].

Tight-binding real space basis simulations have been performed in [4]-[6], where all the circular modes of each carbon ring have been considered at the same time. Such approach can be very demanding from the computational point of view especially for long nanotubes with relatively large diameter.

In case of a device with coaxial symmetry, modes are uncoupled and transport can be computed solving as many independent problems as the number of the considered modes [7]. However, when there is no spatial symmetry, modes are coupled and scattering between modes has to be taken into account. On the other hand, as shown in [7], generally only the lower subbands take part to transport, so that a complete set of modes is not always required.

This work focuses on double-gate CNT-FETs, where the coaxial symmetry of the potential is broken, and on devices with discrete distribution of dopants in the source and drain reservoirs, where any potential symmetry is further reduced. To this purpose a coupled mode space approach has been implemented for the solution of the open boundary condition Schrödinger equation within the NEGF framework, together with the self-consistent solution of the 3-D Poisson equation. Such approach can deal with very general structures and can provide the same results as those yielded by the real space approach, with reduced computational cost, allowing the possibility of performing really computational demanding simulations like those required by a statistical investigation of device performance.

\section{MODEL AND NUMERICAL APPROACH}

The equations for the retarded Green's function $G$ reads [3]

$$
G(E)=\left[\left(E+i \eta^{+}\right) I-H-\Sigma\right]^{-1},
$$

where $E$ is the energy, $I$ the identity matrix, $H$ the Hamiltonian, $\Sigma$ is the boundary self-energy, and $i \eta^{+}$is an infinitesimal imaginary quantity, which takes into account the irreversibility of the considered process. No incoherent scattering processes in the device are considered here. 
Once computed, the Green's function can provide all the relevant transport quantities like for example the electron concentration $n(\vec{r})$ on each carbon site $\vec{r}$. In particular, if $\Sigma_{S}\left(\Sigma_{D}\right)$ and $E_{F_{S}}\left(E_{F_{D}}\right)$ are the self-energy and the Fermi energy of the source (drain), respectively, we have

$$
\begin{aligned}
n(\vec{r})=\frac{1}{\pi} \int d E\left[A_{S}(\vec{r}, E) f(\right. & \left.E-E_{F_{S}}\right) \\
& \left.+A_{D}(\vec{r}, E) f\left(E-E_{F_{D}}\right)\right]
\end{aligned}
$$

where, omitting the dependence on the energy for the considered matrices, the so-called local partial density of states can be expressed as

$$
\begin{aligned}
& A_{S}(\vec{r}, E) \equiv \operatorname{diag}\left\{i G \Gamma_{S} G^{\dagger}\right\}(\vec{r}), \\
& A_{D}(\vec{r}, E) \equiv \operatorname{diag}\left\{i G \Gamma_{D} G^{\dagger}\right\}(\vec{r})
\end{aligned}
$$

where diag is the operator which extracts the diagonal of the matrix to which it is applied, $f$ is the Fermi-Dirac occupation factor, $\Gamma_{S}$ is a $N_{C} \times N_{C}$ block matrix, whose top-left block is the only nonzero one and equal to $\Sigma_{S}$, and $\Gamma_{D}$ is a $N_{C} \times N_{C}$ block matrix, whose bottom-right block is the only nonzero one and equal to $\Sigma_{D}$.

The transmission coefficient $\mathcal{T}$ can be derived as

$$
\mathcal{T} \equiv-\operatorname{Tr}\left[\left(\Sigma_{S}-\Sigma_{S}^{\dagger}\right) G\left(\Sigma_{D}-\Sigma_{D}^{\dagger}\right) G^{\dagger}\right]
$$

where $\operatorname{Tr}$ is the trace operator.

We consider zig-zag nanotubes, starting from the theoretical background proposed by Guo et al. [7] for devices with coaxial geometry, and extending it to devices where any potential symmetry is broken.

If $n$ is the number of atoms on each carbon ring, and $N_{C}$ is the number of carbon rings, the real space Hamiltonian is a tridiagonal block matrix [7], with $N_{C}$ matrices of order $n$.

If $t$ is the hopping parameter, $H$ reads

$$
H=\left(\begin{array}{ccccccc}
D_{1} & \alpha & & & & & \\
\alpha & D_{2} & \beta & & & & \\
& \beta^{\dagger} & D_{3} & \alpha & & & \\
& & \alpha & D_{4} & \beta^{\dagger} & & \\
& & & \beta & D_{5} & \alpha & \\
& & & & \ddots & \ddots & \ddots \\
& & & & & \alpha & D_{N_{C}}
\end{array}\right)
$$

On the $i$-th carbon ring, the diagonal block matrix reads

$$
D_{i}=\left(\begin{array}{cccc}
\Phi_{i 1} & & & \\
& \ddots & & \\
& & \ddots & \\
& & & \Phi_{\text {in }}
\end{array}\right)
$$

where $\Phi_{i j}$ is the potential on the $j$-th carbon atom on the $i$-th ring, while $\alpha=t I_{n}\left(I_{n}\right.$ is the identity matrix of order $\left.n\right)$ and $\beta$ reads

$$
\beta=\left(\begin{array}{cccc}
t & & & t \\
t & \ddots & & \\
& \ddots & \ddots & \\
& & t & t
\end{array}\right) .
$$

It is then trivial that by reducing the size of the system under investigation, and hence reducing the size of the Hamiltonian, one gains benefits from a computational point of view, since most of the computational cost is represented by the partial matrix inversion required in (1). An efficient algorithm for matrix inversion is represented by the recursive Green's function algorithm, which has a complexity $O\left(N_{C} \times n^{3}\right)$ [8], that can be prohibitive if $n$ is large. However, as shown in [7], only the lowest subbands take part to transport in CNT-FETs, so that a simulation study based on a limited number of subbands would provide faster results as accurate as those obtained with a real space approach. From a mathematical point of view, this can be achieved by means of a basis transformation. If $T$ is an unitary operator $\left(T^{\dagger} T=T T^{\dagger}=I\right)$, starting from (1) one obtains

$$
G(E)\left[\left(E+i \eta^{+}\right) I-H-\Sigma\right]=I
$$

and multiplying both sides by $T$ and $T^{\dagger}$

$$
\begin{aligned}
T^{\dagger} G(E)\left[\left(E+i \eta^{+}\right) I-H-\Sigma\right] T & =T^{\dagger} T=I, \\
T^{\dagger} G(E) T T^{\dagger}\left[\left(E+i \eta^{+}\right) I-H-\Sigma\right] T & =I .
\end{aligned}
$$

One finally writes

$$
G^{\prime}(E)=\left[\left(E+i \eta^{+}\right) I-H^{\prime}-\Sigma^{\prime}\right]^{-1}
$$

where

$$
\begin{aligned}
G^{\prime}(E) & \equiv T^{\dagger} G(E) T \\
H^{\prime} & \equiv T^{\dagger} H T \\
\Sigma^{\prime} & \equiv T^{\dagger} \Sigma T .
\end{aligned}
$$

From (12) one can see that the transformed Green's function is just the Green's function corresponding to the transformed Hamiltonian and the self-energy.

$T$ can be a diagonal block matrix $T$ as

$$
T=\left(\begin{array}{cccc}
\tilde{T} & & & \\
& \ddots & & \\
& & \ddots & \\
& & & \tilde{T}
\end{array}\right)
$$

where $\tilde{T}$ is the eigenfunction matrix of the $\beta$ block matrix. In this way, $H^{\prime}$ is still a tridiagonal block matrix, where the upper and the lower block matrices are diagonal, while

$$
D_{i}^{\prime}=\tilde{T}^{\dagger} D_{i} \tilde{T}
$$

As demonstrated in [7], if the considered device has a coaxial geometry, then $D_{i}^{\prime}$ is also diagonal and $H^{\prime}$ can be reordered, so that (1) is reduced to the inversion of a separate $N_{C} \times N_{C}$ matrix for each mode. One then can easily choose how many modes are to be considered. Such property is a particular case of a more general property. Indeed, if the potential term in the Schrödinger equation can be written as

$$
V(x, y, z)=V_{1}(x, y)+V_{2}(z)
$$

i.e., the potential on each $x-y$ plane is equal to $V_{1}(x, y)$ except for a constant value $\left(V_{2}(z)\right)$, it is trivial to demonstrate that the 3 -D Schrödinger equation can be decoupled in a 2-D equation in the plane of confinement, and in a 1-D equation along the 
transport direction. Indeed, the same set of eigenfunctions are found on each $x-y$ plane independent of $z$. The external potential for the 1-D equation is $V_{2}(z)$, shifted by the eigen energy values computed in the $x-y$ plane. The coaxial geometry case for CNTs is a particular case of (18) when $V_{2}$ is constant.

In realistic devices (18) hardly holds, hence $D_{i}^{\prime}$ in (17) is not diagonal. Matrix reduction can, however, still be obtained, considering $\tilde{T}$ as an incomplete orthonormal basis set, whose columns are the first $N_{m}$ eigenfunctions of $\beta$. A matrix $T$ can then be defined, whose dimensions are $\left(n N_{C}\right) \times\left(N_{m} N_{C}\right)$. In this way, the tridiagonal Hamiltonian $H^{\prime}$ has $N_{C}$ diagonal block matrices of order $N_{m}$ along the diagonal. As a consequence, starting from a $\left(n N_{C}\right) \times\left(n N_{C}\right)$ matrix, we have obtained a $\left(N_{m} N_{C}\right) \times\left(N_{m} N_{C}\right)$ matrix, with $N_{m} \leq n$.

Once obtained, the self-consistency between the potential derived from the Poisson equation and the charge density computed by means of the NEGF formalism as in (2), the local partial density of states in the mode-space basis set has to be mapped back to the real space by means of an antitransformation, in order to compute the charge in correspondence of each carbon site. In particular

$$
\begin{aligned}
& A_{S}(\vec{r}, E) \equiv \operatorname{diag}\left\{i T G^{\prime} \Gamma_{S}^{\prime} G^{\prime \dagger} T^{\dagger}\right\}(\vec{r}), \\
& A_{D}(\vec{r}, E) \equiv \operatorname{diag}\left\{i T G^{\prime} \Gamma_{D}^{\prime} G^{\prime \dagger} T^{\dagger}\right\}(\vec{r}) .
\end{aligned}
$$

The reduction of the basis to a subset of modes $\left(N_{m} \leq n\right)$, while introducing an approximation to the method, has the advantage, as demonstrated above, of reducing the complexity of the problem, and hence the computing time. However, as we will demonstrate in the following sections, such an approximation provides results in perfect agreement with those derived by the real space approach, since only few modes effectively take part to transport.

\section{A. Self-Energy Computation}

From a numerical point of view, particular attention must be put in the definition of a fast and reliable procedure for the computation of the self-energy $\Sigma$, which, in the NEGF formalism, can be considered as the boundary condition for the Schrödinger equation. As in [9], $\Sigma$ reads

$$
\Sigma=\tau g \tau^{\dagger}
$$

where $\tau$ is the so-called coupling matrix between the reservoirs and the channel, while $g$ is the surface Green's function, which is computed recursively. The key point in the self-energy computation is then finding $g$. Different methods can be used in order to accomplish this task. The simplest method is the under-relaxation method, which has serious convergence problems, especially for energies in correspondence of Van-Hove singularities [10]. Such problems can be avoided by means of the transfer Hamiltonian formalism as proposed by Sancho et al. [10], which works better in correspondence of the singularities, and, for each energy point, $g$ is found with almost the same number of iterations (about 15). However, if the potential at the CNT ends can be considered constant, the self-energy can be computed in a closed form, by means of an analytical expression [7]. In Fig. 1 the time needed to compute the Green's function $G$ on 100 energy points and for $N_{C}=6$, with the three above-mentioned

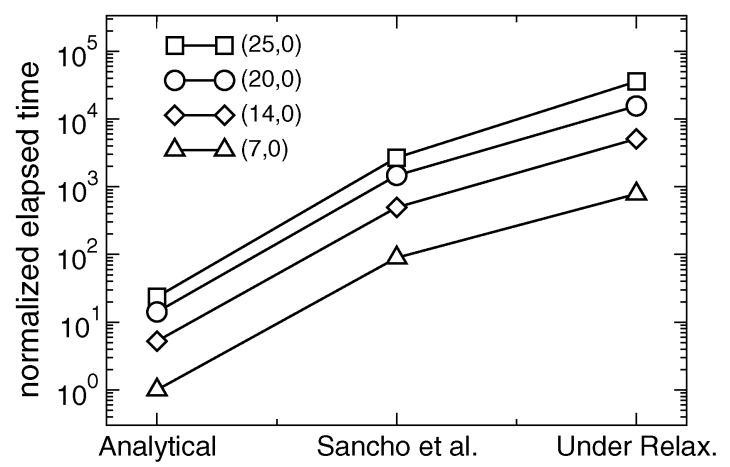

Fig. 1. Time required to compute the Green's function $G$ when different methods are considered to compute the self-energy, for different chiralities. The results are normalized with respect to the fastest results (analytical and $(7,0)$ nanotube).

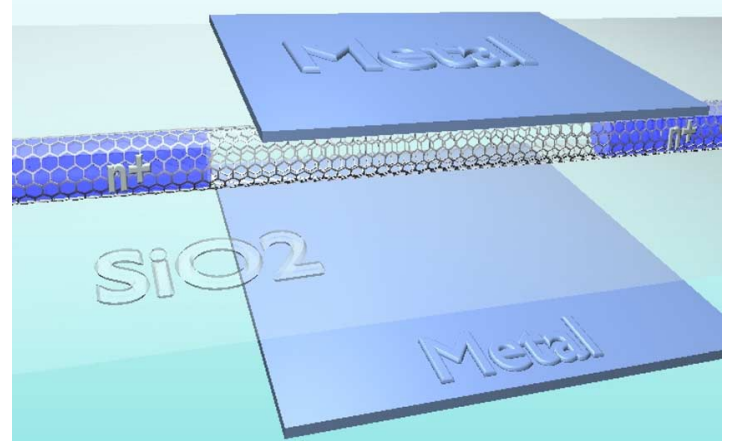

Fig. 2. 3-D structure of the simulated double-gate CNT-FET.

methods and for different carbon nanotube chiralities is shown. As can be seen, the approach in [10] is always 8 times faster than the under-relaxation method, while the analytical method is almost 700 times faster than the under-relaxation method.

\section{RESULTS AND DisCUSSIONS}

The performance of the coupled mode space approach for the simulation of CNT-FET devices is evaluated. Double-gate CNT-FETs, and devices in which doping impurities are distributed randomly in the source and drain reservoirs are considered. In both cases, the potential is not constant along each carbon ring, especially for the random dopant distribution case, where spikes in correspondence of ionized impurities appear. In realistic devices the cylindrical geometry is broken and potential is not constant on the same carbon ring. Modes become coupled, hence a coupled mode space approach is required in order to recover quantitative results.

\section{A. Double-Gate CNT-FET}

In Fig. 2 the considered 3-D structure is shown. Simulations have been performed solving the Poisson equation in the 3-D domain self-consistently with the Schrödinger equation within the NEGF formalism on each carbon atom. In Fig. 3(a), the transfer characteristic of a $(10,0)$ CNT-FET is shown for a drain-tosource voltage $V_{\mathrm{DS}}=0.1 \mathrm{~V}$ computed both with the real and the mode space approach, when two modes are considered. As 


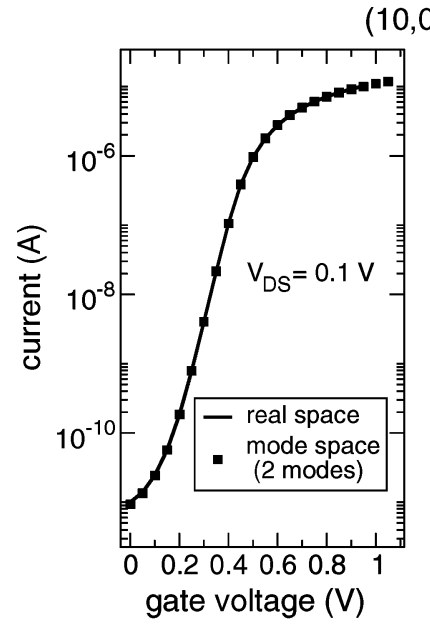

(a)

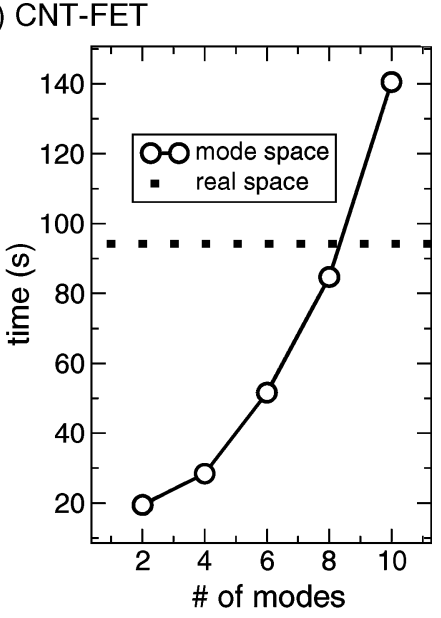

(b)
Fig. 3. (a) Transfer characteristic and (b) execution time on an AMD 64 bit processor as a function of the number of modes for a $(10,0)$ DG CNT-FET.

$(25,0)$ CNT-FET

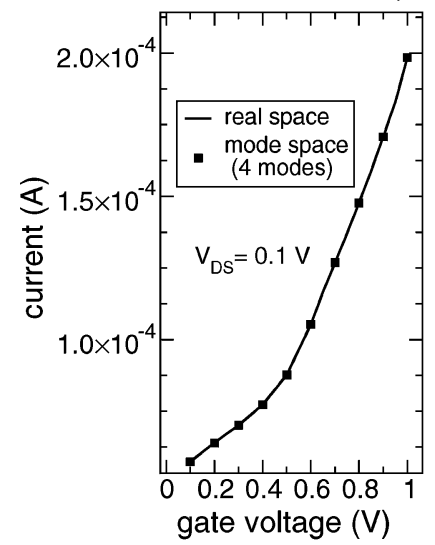

(a)

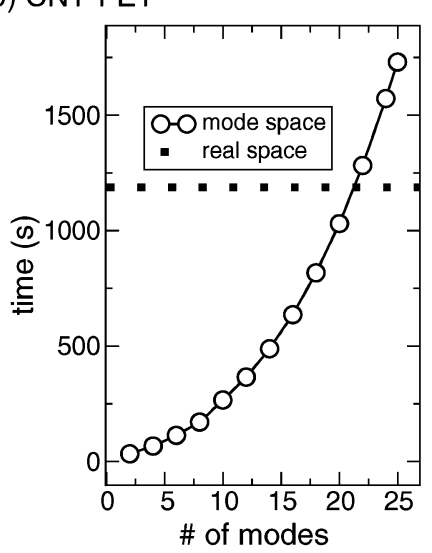

(b)
Fig. 4. (a) Transfer characteristic and (b) execution time on an AMD 64 bit processor as a function of the number of modes for a $(25,0)$ DG CNT-FET.

can be seen, results perfectly match, but with very different execution time. We plot in Fig. 3(b) the time needed by a $2.0 \mathrm{GHz}$ AMD 64 bit processor to perform the NEGF simulation on 2800 atoms as a function of the number of modes: the higher the number of modes, the larger the computational cost. Due to degeneracy of CNT band structure, we consider only even number of modes. Simulating time increases with the number of modes. When ten modes are considered, the mode space approach is slower than the real space approach, since a basis transformation has to be performed on top of all the operations needed by the real space approach. However, when only two modes are considered, the mode space approach is five times faster.

In Fig. 4(a) and (b) the same kind of results are shown for a $(25,0)$ CNT-FET. In this case, more modes (four modes) are needed in order to accurately reproduce the transfer characteristics provided by the real space approach, because of the larger diameter. When four modes are taken into account, the mode space is almost 20 faster than the real space approach. A safe approach to choose the number of considered modes for a given

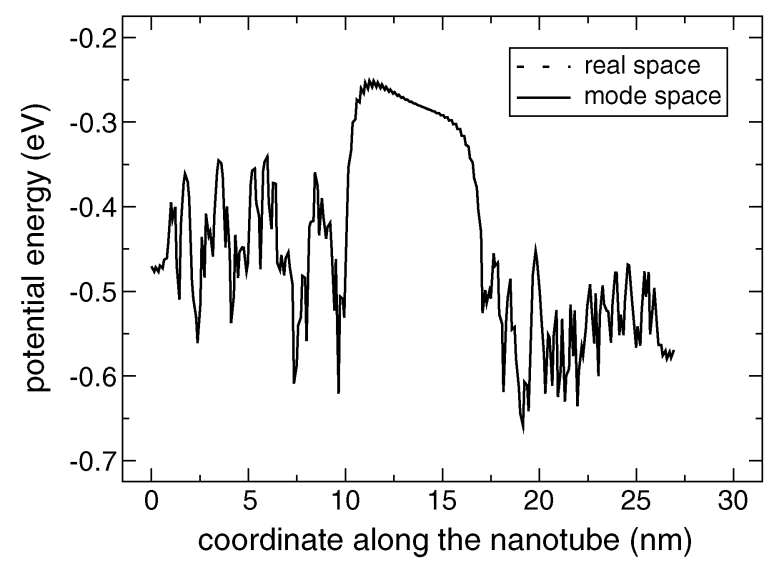

Fig. 5. Average of the potential energy on the carbon ring computed by means of the real and mode space approach for a $(13,0)$ CNT-FET where ionized impurities are present.

structure and bias is to perform multiple simulations with increasing number of modes, and then stop when adding more modes does not modify the obtained results in a significant way.

\section{B. Discrete Ionized Impurities}

The advantage of adopting a mode space approach is apparent when a statistical investigation is performed. In this section we focus on the effects of a random distribution of dopants in the source and drain extensions on the threshold voltage. The mode space approach has been followed, taking into account only the lowest two circular modes, and considering a uniform doping at the CNT ends in order to assure constant potential at the boundaries, so to retain the analytical formula for the self-energy.

Fig. 5 plots the average potential energy on the carbon ring computed both with the real and mode space approach for a $(13,0)$ CNT-FET. We want to stress the fact that even in the case of a very rough potential, the two different approaches provide the same results, while, in this case, mode space approach is at least six times faster than the real space approach.

From a numerical point of view, we have specified in each reservoir a nominal donor fraction $f$ and the ionization fraction

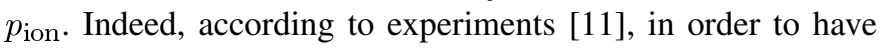
stable doped CNTs, functional groups have to be preferred to alkali group. Such a solution leads to partially ionized impurities, as it happens when other molecules like $\mathrm{NH}_{3}$ are used, as demonstrated experimentally by [12], where 0.04 electrons per molecule are donated to the CNT. In particular, in order to simulate the actual distribution of dopants in the source and drain extensions, starting from the nominal donor and ionization fractions, a random variable $N^{\prime}$ uniformly distributed in the interval $[0,1]$ has been extracted in correspondence of each carbon site: if $N^{\prime}$ is smaller than $f$, then a positive fixed charge equal to $p_{\text {ion }}$ is imposed on the carbon site, otherwise the fixed charge is zero.

In this way, simulating a large number of devices with the same nominal doping profile, but with a different actual distribution of charge, we have been able to investigate the threshold voltage dispersion.

In the considered devices, the number of ionized impurities is of the order of few tens. The threshold voltage $V_{T}$, defined as the gate voltage at which the current is equal to $0.1 \mu \mathrm{A}$, can then be sensitive to intrinsic dopant fluctuations. 


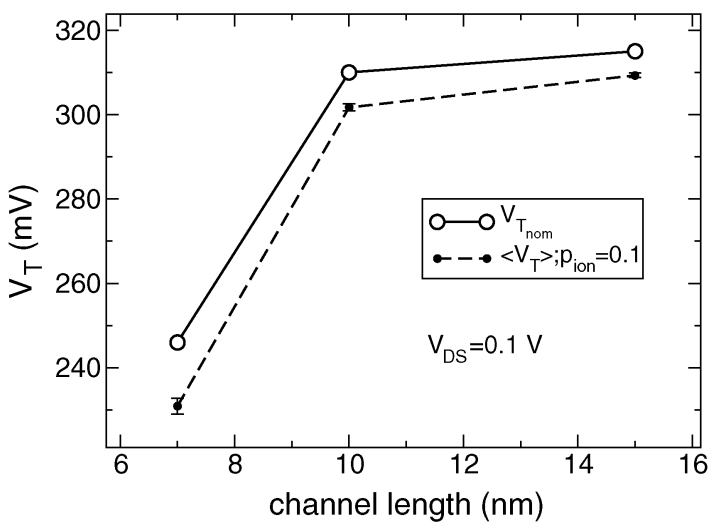

Fig. 6. Nominal threshold voltage $V_{T_{\text {nom }}}$ computed with uniform doping in the reservoirs and mean threshold voltage computed for $p_{\text {ion }}=0.1$ as a function of the channel length.

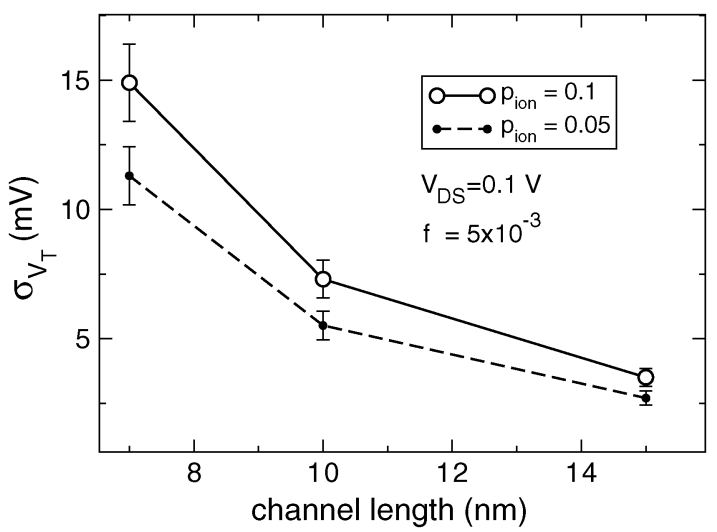

Fig. 7. Standard deviation of the actual threshold voltage $V_{T}$ and $V_{T_{n o m}}$, as a function of the channel length and for two different values of $p_{\text {ion }}$, and the same average number of donated electrons $\left(5 \times 10^{-3}\right)$.

A statistical analysis on the threshold voltage has been performed on an ensemble of 50 nominally identical devices, but with different actual doping profile. The considered number of simulated devices is a good tradeoff between accuracy and computational cost, providing a standard deviation of the error on the second order moment of $\sigma_{V_{T}} / \sqrt{2 M}$, where $M$ is the sample size, and $\sigma_{V_{T}}$ is the standard deviation of the threshold voltage $V_{T}$.

In Fig. 6 the nominal threshold voltage $V_{T_{\text {nom }}}$ computed for the three different devices in the case of uniform doping and for a drain-to-source voltage $V_{\mathrm{DS}}=0.1 \mathrm{~V}$ is shown, as well as the mean threshold voltage computed for $p_{\text {ion }}=0.1$.

As the channel length is decreased, the threshold voltage decreases, because of short channel effects.

Fig. 7 shows $\sigma_{V_{T}}$ for two different values of $p_{\text {ion }}$, but with the same average number of donated electrons $\left(5 \times 10^{-3}\right)$ : as the channel length is increased, the standard deviation decreases, since short channel effects become less relevant, and potential fluctuations in the source and in the drain do not influence the potential in the channel. In addition, larger $p_{\text {ion }}$ determines larger dispersions, which, however, are always smaller than $6 \%$ of $V_{T_{\text {nom }}}$, as shown in Table I. In the same table, the standard deviation of the threshold voltage $\sigma_{V_{T_{s}}}\left(\sigma_{V_{T_{d}}}\right)$ is shown, defined as the standard deviation when random doping is imposed in the source (drain) region, while uniform doping in the drain
TABLE I

STANDARD DEVIATION OF THE THRESHOLD VOLTAGE: $\sigma_{V_{T_{s}}}\left(\sigma_{V_{T_{d}}}\right)$ IS THE STANDARD DEVIATION WHEN RANDOM DOPING IS IMPOSED IN THE SOURCE (DRAIN) AND UNIFORM DOPING IN THE DRAIN (SOURCE) EXTENSION, COMPUTED FOR $p_{i o n}=0.05$ AND FOR $p_{i o n}=0.1$

\begin{tabular}{|c|c|c|c|c|c|c|}
\hline$p_{\text {ion }}$ & $\begin{array}{c}\mathrm{L} \\
(\mathrm{nm})\end{array}$ & $\begin{array}{c}\sigma_{V_{T_{s}}} \\
(\mathrm{mV})\end{array}$ & $\begin{array}{c}\sigma_{V_{T_{d}}} \\
(\mathrm{mV})\end{array}$ & $\begin{array}{c}\frac{\sigma_{V_{T_{s}}}}{V_{T}} \\
(\%)\end{array}$ & $\begin{array}{c}\frac{\sigma_{V_{T_{d}}}}{V_{T}} \\
(\%)\end{array}$ & $\begin{array}{c}\frac{\sigma_{V_{T}}}{V_{T}} \\
(\%)\end{array}$ \\
\hline 0.05 & 7 & 6.3 & 8.7 & 2.56 & 3.54 & 4.59 \\
\hline 0.05 & 10 & 3.7 & 2.9 & 1.189 & 0.932 & 1.77 \\
\hline 0.05 & 15 & 2.3 & 1 & 0.73 & 0.317 & 0.857 \\
\hline 0.1 & 7 & 9 & 11.9 & 3.66 & 4.84 & 6 \\
\hline 0.1 & 10 & 5.2 & 5.1 & 1.67 & 1.639 & 2.3 \\
\hline 0.1 & 15 & 3.5 & 1.6 & 1.11 & 0.507 & 1.175 \\
\hline
\end{tabular}

(source) extensions, computed for $p_{\text {ion }}=0.05$ and $p_{\text {ion }}=0.1$, respectively.

For the shortest devices at small $V_{\mathrm{DS}}=100 \mathrm{mV}, \sigma_{V_{T_{s}}}$ and $\sigma_{V_{T_{d}}}$ are comparable, since fluctuations of the potentials at the source and drain extensions affect both the potential barrier in the channel (short channel effects), and the backscattering probability of electrons propagating from the source.

\section{CONCLUSION}

A coupled mode space approach has been proposed and implemented for the simulation of CNT field effect transistors with no spatial symmetry. The mode space approach reduces the computational load by typical factors of 4-6 compared to the real space approach. This enables one to perform statistical investigations such as analysis of the threshold voltage dispersions due to the random distribution of ionized impurities in the source and drain reservoirs. Particular attention must be put in the choice of a proper iterative method for the self-energy computation, if fast results are to be achieved.

\section{REFERENCES}

[1] S. O. Koswatta, N. Neophytou, D. Kienle, G. Fiori, and M. S. Lundstrom, "Dependence of DC characteristics of CNT MOSFETs on bandstructure models," IEEE Trans. Nanotechnol., vol. 5, no. 4, pp. 368-372, Jul. 2006.

[2] M. Pourfath, E. Ungersboek, A. Gehring, B. H. Cheong, and W. Park et al., "Improving the ambipolar behavior of schottky barrier carbon nanotube field effect transistors," in Proc. ESSDERC 2004, pp. 429-432.

[3] S. Datta, "Nanoscale device modeling: The Green's function method," Superlattices Microstruct., vol. 5, pp. 253-278, 2000.

[4] J. Guo, A. Javey, H. Dai, and M. Lundstrom, "Performance analysis and design optimization of near ballistic carbon nanotube field-effect transistors," in IEDM Tech. Digest, 2004, pp. 703-706.

[5] G. Fiori, G. Iannaccone, and G. Klimeck, "A three-dimensional simulation study of the performance of carbon nanotube field-effect transistors with doped reservoirs and realistic geometry," IEEE Trans. Electron Devices, vol. 53, no. 8, pp. 1782-1788, Aug. 2006.

[6] D. L. John and D. L. Pulfrey, "Real-performance modeling of carbon nanotube FET," in Proc. IWCE-11, 2006, pp. 253-254.

[7] J. Guo, S. Datta, M. Lundstrom, and M. P. Anantram, "Multi-scale modeling of carbon nanotube transistors," Int. J. Multiscale Comput. Eng., vol. 2, pp. 257-260, 2004

[8] R. Lake, G. Klimeck, R. C. Bowen, and D. Jovanovic, "Single and multi-band modeling of quantum electron transport through layered semiconductors devices," J. Appl. Phys., vol. 81, pp. 7845-7869, Feb. 1997.

[9] P. S. Damle, A. W. Gosh, and S. Datta, "Unified description of molecular conduction: From molecules to metallic wires," Phys. Rev. B, vol. 64, p. $201403,2001$.

[10] M. P. L. Sancho, J. M. L. Sancho, and J. Rubio, "Highly convergent schemes for the calculation of bulk and surface green function," J. Phys. F, Met. Phys., vol. 15, pp. 851-858, 1985 
[11] M. Shim, A. Javey, N. W. S. Kam, and H. Dai, "Polymer functionalization of air-stable n-type carbon nanotube field effect transistors," $J$. Amer. Chem. Soc., vol. 123, pp. 11512-11513, 2001.

[12] K. Bradley, J. P. Gabriel, M. Briman, A. Star, and G. Grüner, "Charge transfer from ammonia physisorbed on nanotubes," Phys. Rev. Lett., vol. 91, pp. 218301-1-218301-4, 2003.

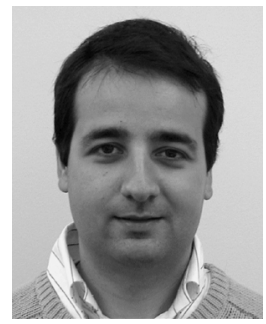

Gianluca Fiori received the degree in electronic engineering and the $\mathrm{Ph}$. D. degree from the University of Pisa, Italy, in 2001 and 2005, respectively.

In autumn 2002, he was in SILVACO International developing quantum models, which are currently implemented in the commercial simulator ATLAS by SILVACO. In summer 2004 and 2005, he was at Purdue University, working on models for the simulation of transport in nanoscale devices. He is currently working at Dipartimento di Ingegneria dell'Informazione, at Pisa University, holding a postdoc position. His main field of activity is the development of models and codes for the simulations of ultrascaled semiconductor devices. Further information is available at http://www.monteverdi.iet.unipi.it/ fiori.

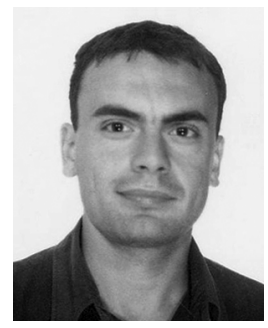

Giuseppe Iannaccone received the M.S. and Ph.D degrees in electrical engineering from the University of Pisa, Italy, in 1992 and 1996, respectively.

In 1996 he took a permanent position as researcher with the Italian National Research Council and the same year he obtained a faculty position in the Electrical Engineering Deparment of the University of Pisa, first as an Assistant Professor, then, since January 2001, as Associate Professor of Electronics. $\mathrm{He}$ has authored and coauthored more than 110 papers published in peer-reviewed journals and more than 70 papers in proceedings of international conferences. His interests include transport and noise in nanoelectronic and mesoscopic devices, development of device modeling and TCAD tools, and the design of extremely low power circuits and systems for RFID and ambient intelligence scenarios.

Prof. Iannaccone has coordinated a few European and national projects involving multiple partners, and has acted as principal investigator in several research projects funded by public agencies at the European and national level, and by private organizations. He is in the technical committee of few international conferences and serves as a referee for the leading journals in the fields of condensed matter physics, device electronics, and circuit design.

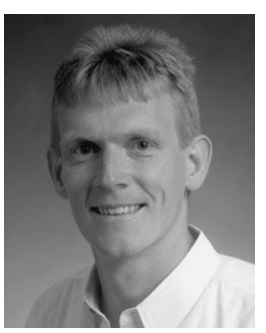

Gerhard Klimeck (S'94-M'96-SM'04) received the German electrical engineering degree from Ruhr-University Bochumin 1990 and the Ph.D. degree from Purdue University, West Lafayette, IN, in 1994.

Previously he was a member of technical staff at the Central Research Lab of Texas Instruments. He was the Technical Group Supervisor for the Applied Cluster Computing Technologies Group and continues to hold his appointment as a Principal Member at the NASA Jet Propulsion Laboratory on a faculty part-time basis. He is currently the Technical Director of the Network for Computational Nanotechnology and Professor of Electrical and Computer Engineering at Purdue University. He leads the development and deployment of Web-based simulation tools that are hosted on http://www.nanohub.org, a community Web site that is utilized by over 21000 users annually. His work is documented in over 170 peer-reviewed publications and over 260 conference presentations. His research interest is in the modeling of nanoelectronic devices, parallel cluster computing, and genetic algorithms. He has been the lead on the development of NEMO 3-D, a tool that enables the simulation of tens-of-million-atom quantum dot systems, and NEMO 1-D, the first nanoelectronic CAD tool.

Dr. Klimeck is a member of APS, HKN and TBP. More information can be found at http://www.ece.purdue.edu/ gekco and http://www.nanoHUB.org/ klimeck. 